\title{
Methods for determining the number of closed cells in rigid sprayed polyurethane foam
}

\author{
Marina V. Gravit ${ }^{1 *}$, Olamipe Ogidan ${ }^{1}$, and Elena Znamenskaya ${ }^{2}$ \\ ${ }^{1}$ Peter the Great St. Petersburg Polytechnic University, 195251 St. Petersburg, Russia \\ ${ }^{2}$ Moscow State University of Civil Engineering, Yaroslavskoe shosse, 26, Moscow, 129337, Russia
}

\begin{abstract}
Polyurethane foams are currently the most effective thermal insulation materials, poly isocy anurates have low combustibility. Predicting the number of closed cells forming a gas-filled matrix in the finished polymer is necessary to improve the quality and durability of rigid sprayed polyurethane foam (SPF) and also be able to determine their quantity in finished coatings using standardized techniques. Depending on the number of closed cells, the polyurethane coatings are classified into certain levels in European documents. The methods of determining the volume percentage of open and closed cells of polyurethane foam, the problems of applying these methods, measuring equipment and their errors are analyzed in the article. The authors analyzed the international standard ISO 4590 for determining the volume percentage of open and closed cells spread in the EU countries and the American standard ASTM D 6226 for the purposes of harmonization of European standards with the developed national standard for PUR insulation and polyisocyanurate (PIR), sprayed on the site of production.
\end{abstract}

\section{Introduction}

In Russia, for rigid sprayed polyurethane foam, two national standards are being developed, one of which is GOST R "Thermal insulation coating of polyurethane foam (PUR) and polyisocyanurate foam (PIR), sprayed on the site of production. Technical Conditions", the provisions of the most effective methodology and the recommended type of equipment for determining the number of closed cells in polyurethane foams will be included. To improve the quality and durability of rigid SPF and the overall understanding of the provision of most technological characteristics, it is necessary to predict the number of closed cells forming a gas-filled matrix in the finished polymer, be able to determine their quantity in finished coatings (as well as the dimensions, wall thickness of cells etc.), using standardized methods [1].

Table 1 shows the classification, depending on the number of closed cells, of the content of open and closed cells in the British version of the European standard BS EN 14315-1: 2013 - Thermal insulating products for buildings - In-situ formed sprayed rigid polyurethane (PUR) and polyisocyanurate (PIR) foam products. Part 1: Specification for the rigid foam spray system before installation (PUR) and [1]:

*Corresponding author: marina.gravit@mail.ru 
Table 1. Closed-cell content classification.

\begin{tabular}{|c|c|}
\hline Class & Content of closed cells, $\%$ \\
\hline $\mathrm{CCC} 1$ & $<20 \%$ \\
\hline $\mathrm{CCC} 2$ & $20 \%$ to $80 \%$ \\
\hline $\mathrm{CCC} 3$ & $>80 \%$ to $89 \%$ \\
\hline $\mathrm{CCC} 4$ & $>90 \%$ \\
\hline
\end{tabular}

Current research on rigid polyurethane foam with various foaming agents is focused on diffusion and sorption of water vapor and their relationship to the thermo-physical properties of the resulting material. Much research been devoted to these studies, for example [2 - 5]. Moisture absorption in samples foamed with freons is generally lower than for samples foamed with $\mathrm{CO} 2$, however, according to studies [3], after 1200 hours of moisture absorption, a significant increase in the thermal conductivity (about $15 \%$ on average for each sample), which confirms rapid diffusion of air for a noted period of time in the cells unpressurized by the final coating of the polyurethane foam.

The classification in the cited standard [1] is important for determining the thermal conductivity in the processes of operation (aging) (it is listed in the Annex J "Method for the presentation of thermal conductivity/thermal resistance). According to the text [1], factoryproduced and site produced polyurethane sprayed with CCC4 contain more than $90 \%$ of closed cells and foaming agents, which are stored in the cells long enough. As mentioned above, the initial thermal conductivity of such products is lower than in products where the cells contain air; destruction takes place when air enters by diffusion into closed cells, which increases the thermal conductivity of the resulting gas mixture in the cell, unless this is prevented by applying special surface (finish) coatings that prevent diffusion.
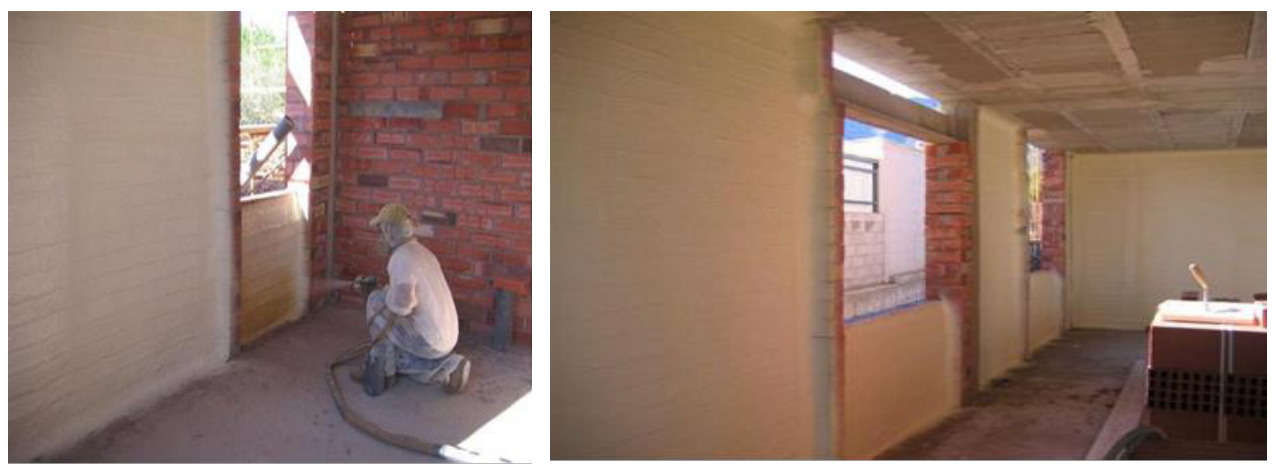

Fig. 1 - Application of poly urethane foam for thermal insulation

On the other hand, polyurethane foam with open cells, thermal conductivity is practically the same in the aging process, since air is initially present in the product. Polyurethane and $\mathrm{CCCl}$ polyisocyanurate with a closed cell content below $20 \%$ belong to this category, because only a very small fraction of the cells can be responsible for the aging process only if the internal diffusion of air is not prevented by the application of a protective coating. However, their initial thermal conductivities are higher than those of CCC4 products, since the products ofCCC4 contain initially a small amount of air. 
It follows that the increase in thermal conductivity caused by aging is due to diffusion of air (if not prevented), for products with intermediate classes of closed cells, that is, for products in classes CCC 3 and $\mathrm{CCC}$, will be less than the value for products in the CCC4 class, but more than products in the $\mathrm{CCCl}$ class. Therefore, their initial values are between the values of class es CCC4 and CCC1. Since the aging process is caused by air diffusion, the effect on thermal conductivity depends on the thickness, since the outer cells must create a partial pressure of the air gases before starting the transfer to the cells of the next layer. The aging procedure at high temperature, described in Appendix $C$, is designed to simulate the degree of aging corresponding to averaged a period of operation for 25 years to obtain a value close to the real one for calculating the heat loss for the product during its service life. Accordingly, for example, for prefabricated products and especially for CCC4 products used in buildings, the value after aging depends on the thickness of the product, while the value for the $\mathrm{CCC} 1$ class is less dependent on the thickness. For products of class CCC4, since the value of thermal conductivity after aging depends strongly on the thickness of the product, and also whether there is any diffusion-preventing finish in the final application of the product, it is necessary to indicate the thermal resistance according to the thickness under three special conditions with respect to any surfaces that prevent diffusion. For products of class $\mathrm{CCC1}$, in the absence of closed cells there will be no aging, so thermal resistance will simply be a function of thickness. Accordingly, the manu facturer can decide that it is possible not to take into account the presence or absence of any surfaces in the final application of the diffusion-preventing material. The solution depends on the level of the content of the closed cells of the product, which by definition can not be more than $20 \%$. For products of class CCC2 and CCC3, these materials have a closed cell content of $20 \%$ to $89 \%$, so they require characteristic diagrams based on the example diagrams for class CCC4. However, the degree of dependence of the final value of the thermal resistance after aging on the thickness will be much smaller than for the class CCC4.

Methods of accelerated aging, specified in subparagraphs C.4 and C.5 in EN 14315-1 are primarily developed for PUR/PIR products with closed cell content of greater than or equal to $90 \%$ and which are manufactured using high molecular weight blowing agents such as HFC 134a, 245fa, 227ea, 365mfc, which remain in the cells of the coating during the period specified by the manufacturer and more [1].

In the American standards for rigid polyurethane foam, the classification according to the percentage of closed cells is absent, this parameter is given as one of the physical propert ies (see Chapter 3 of this NTO).

\section{Methods}

Determination of the number of closed and open cells in the regulatory framework of the world is currently produced according to two standards: ISO 4590 Rigid cellular plastics Determination of the volume percentage of open cells and ASTM D6226-15 Standard Test Method for Open Cell Content Rigid Cellular Plastics.

European regulatory documents use the methods set out in ISO 4590 [6] while the American use the method set out in ASTM D6226 [7].

Standard ISO 4590 contains two methods for determining open cells: the method of changing pressure and the method of expanding the volume. The first method is similar to the method in ASTM D6226 [2,7], but differs in the preparation of the experiment and the number of samples [7] (see Table 14).

The determination of volume is based on the application of the Boyle-Mariotte law to the gas compressed in a non-deformable chamber, first without a test sample, and then in the presence of a test sample. We use the most simplified scheme for understanding the 
calculation of actual density, using the same principle as for the determination of the number of cells.

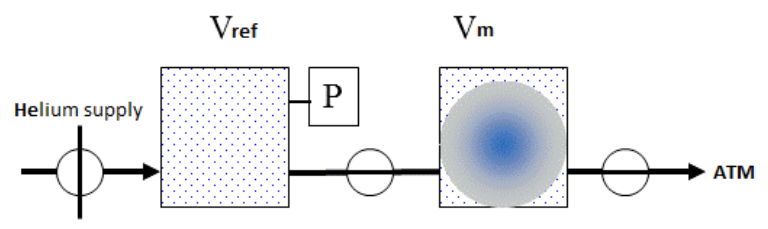

Fig. 2 - General scheme of the principle of the gas pycnometer

The pycnometer consists of two interconnected chambers, a reference and a measuring chamber, equipped with temperature and pressure sensors. Initially, the reference chamber of a known volume, designated in the Vref scheme, is filled with helium, then, when the connecting valve is opened, the gas moves to a measuring chamber of a known volume, designated $\mathrm{Vm}$, into which is placed a sample of a solid material whose density is to be measured. During the experiment, the pressure sensor readings are taken, the equations of material balance are compiled, from which it is not difficult to obtain the volume of the material understudy, knowing the mass and volume of the sample, calculate the true density. For comparison, the scheme of a gas pic-manometer is given in [6] and [7].

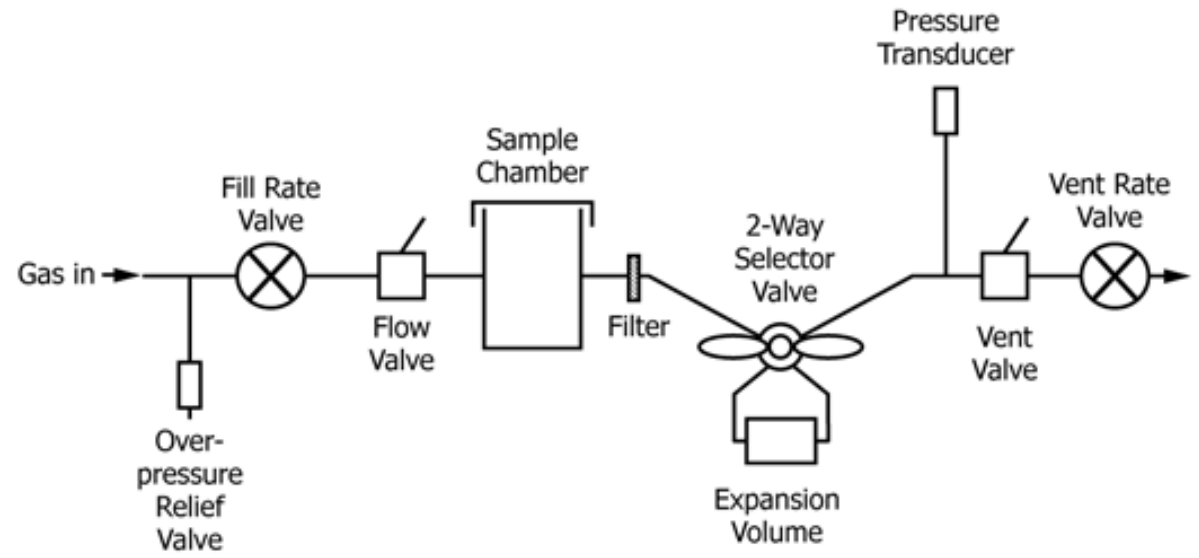

Fig. 3 - Scheme of operation of a gas pycnometer according to ASTM D6226-15

The test method according to ASTM D 6226-15 specifies numerical values for open and closed cells, porous materials. Since any sample is usually obtained by cutting, some of the closed cells will be opened, so they will be included as open cells. One method is used to measure the impermeable volume. ISO Standard 4590-2016 establishes a general procedure for determining the volume percentage of open and closed pores of rigid porous plastics by first measuring the geometric volume and then the airtight volume of the test pieces.

The procedure involves adjusting the apparent volume of open pores, taking into account surface pores opened by cutting during sample preparation. Three alternative methods are provided for measuring impermeable volume. 


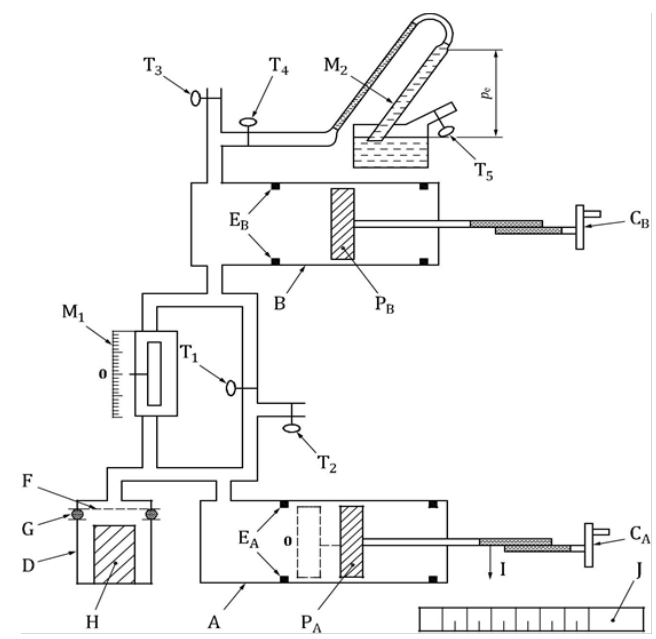

Fig. 4 - Scheme of operation of a gas pycnometer according to ISO 4590

ASTM D 6226-15 defines the porosity based on measuring the available geometric volume of the material, then the volume occupied by the closed cells and the cell walls. To measure the impermeable volume, one method is provided using a pycnometer, which consists of two chambers of a known volume connected by a valve. The chamber calibrated for the samples is available for insertion of the test sample and connected to a source of dry gases with a high degree of purity (at least $99.99 \%$ ), after which the pressure increases to a certain value; After opening the valve, the second pressure is observed. The change in P1/ $\mathrm{P} 2$ is directly related to the sample size of the camera displaced by the sample. The difference between this volume and the geometric volume of the sample is the volume of open cells. Closed cells and cell walls occupy the remaining volume. The accuracy of this method decreases as the content of closed cells and cell sizes increase.

Three alternative methods for ISO 4590-2016 are provided for measuring impermeable volume. Two methods to change the pressure and one method to expand the volume. The volume is determined first without a test sample, and then in the presence of a test sample. The apparent volume percentage of the open pores of the test sample $\omega r$ is calculated by plotting the curve $\omega r=f(r)$ and extrapolating to $r=0$, and then calculating the corrected volume percentage of the open pores $\omega 0$ and the corrected volume percentage ratio of the closed pores $\psi 0$.

For 1 method, the characteristics of atmpamb and the reduction of pe in the chamber with respect to pamb are established. If the volume $\delta \mathrm{VA} 1$ of the chamber is changed without a test specimen and the corresponding volume change $\delta$ VA 2 of the test chamber is in the presence of the test sample.

Both standards are used to determine the numerical values of open and closed cells of porous materials having cavities or cells. In both standards, the procedure involves adjusting the apparent volume of open pores, taking into account surface pores opened by cutting during sample preparation. The symbols and terms of the definition for the compared standards differ due to differences in testing. Pycnometer, ASTM D6226 and ISO 4590-2016 have design differences (see Fig.4, Fig. 5), hence calibration of pycnometers also differs. There are differences in the form of samples and in test preparation. Exposure of samples for testing varies, but the test conditions are similar. In the pycnometer (ISO 4590), the pressure gauges can exhibit instability, resulting in incorrect measurement. In ISO 4590 there are three conditions for performing tests. Particularly if two conditions are not met and the third indication is still less than the second, new measurements must be made according to the specified procedure. 
From the above sequences of test procedures, it can be concluded that the operating principle of a pycnometer based on ISO 4590 is generally more complex and time-consuming than the principle of a pycnometer based on ASTM D 6226. The procedure for correcting the open cells formed as a result of sample preparation is also the main difference in the above standards.

The correction in ASTM D 6226-15 has two correction procedures for open cells during sample preparation. In the first procedure, sample calculations are carried out for only two cubes and will not apply to samples of cylinders. Geometric area of the surface A is calculated by summing up the areas of the two components of the sample in square centimeters as follows:

- average chord length, $t$ of material, is determined by the ASTM D3576 test method, volume occupied by the surface cells, Vs, is calculated using the geometric surface area, A and $\mathrm{t}$

- $\quad$ content of open cells of the sample is calculated, Ov

The second corrected procedure is identical to the procedure in ISO standard 4590-2016.

Variant of calculations without considering the correction of open cells. Geometric volume, $\mathrm{V}$ in cubic centimeters of the sample is calculated by determining its dimensions.

The content of open cells Ov for each sample is expressed as a percentage of the calculated volume V.

If required, the percentage volume occupied by closed cells and cell walls, Cwv, is calculated by equation.

When the specific weight of the material in the solid state is known, it is possible to individually calculate the percentage volumes occupied by the cell walls, $w v$ and closed cells, $c v$.

The percentage of the cell wall volume is then calculated after which the percentage of the volume of the closed cell is calculated using the equation.

In the correction to ISO 4590-2016 for the pressure change method, there is one procedure that is identical to the second procedure in ASTM D 6226-15.

After determining $\mathrm{Vi}$ for each of (at least) three samples, it is necessary to cut each sample three times along three middle planes to get eight cubes. Then it is required to determine the impermeable volume of each set of eight cubes and fix the average volume as the value Vd.

From the compared corrected procedures, it can be concluded that ASTM D $6226-15$ has two procedures, one of which is identical to the procedure us ed in ISO 4590-2016.

Considering the studies given in the standards [6], [7] for calculating the error of the method, correctness of its repeatability, and reproducibility, specifically, using the pressure change method (method 1) in ASTM D 6226 -15 and ISO 4590-2016. The accuracy of measuring the percentage of open pores, including the correction, taking into account the surface pores opened during sample preparation, should be characterized by repeatability and reproducibility.

For ISO 4590-2016, the error values using the pressure change method (method 1) are derived from the cyclic test results carried out in 1981 in ASTM systems, 100 Barr Harbor Drive, West Conshohocken, PA 19428, USA [6]. Tests were conducted in five laboratories. Each test result was the average of five samples. Each laboratory provided one result for each material.

Repeatability: When comparing two average values of each sample for the same material, obtained by the same operator using the same equipment on the same day, the average values should not be considered equivalent if the difference between them exceeds the value of Ir for this material.

Reproducibility: When comparing two average values (each of five samples) for the same material obtained by different operators using different types of equipment on different 
days, the average values should not be considered equivalent if the difference between them is greater than the IR value for the material.

Repeatability and reproducibility is only intended to provide a meaningful way to consider the approximate accuracy of the pressure change method.

As a conclusion, it is pointed out that there are no authoritative standards, according to which it is possible to estimate the errors for this test method. Also, the accuracy of method 1 is not established due to the lack of reference materials for the characteristics to be determined. (The accuracy of methods $2 \mathrm{a}$ and $2 \mathrm{~b}$ (change in volume) is unknown in ISO 4590, and the data obtained using these methods should not be used to resolve disputes between suppliers and users).

For ASTM D 6226-15 in 2004, six laboratories for four materials were tested, each laboratory conducted five tests for each material. It is believed that the test results should not be strictly applied to other samples and batches, conditions, etc. It can also be concluded out that there are no authoritative standards, according to which it is possible to estimate the errors for this test method.

In 2016, a new version of ISO 4590-2002, ISO 4590: 2016, Rigid cellular plastics Method 1 is simplified and more approximate to ASTM D6226-15 Standard Test Method for Open Cell Content of Rigid Cellular Plastics.

In Kazakhstan, the national standard ST RK ISO 4590 "Rigid hard plastic. Determination of the volume percentage open and closed pores ratio, which is harmonized as identical with ISO 4590: 2016, that is, all provisions of the standard have been adopted in full (an identical translation has been made).

In pycnometers, nitrogen, krypton, helium, mercury, hydrogen, oxygen, carbon oxides, etc. can be used as a working tool (differing in molecular size). and a relatively new proposal - hollow glass spheres. Manufacturers of equipment in technical information describe the scope of application of devices either too short, or too broad (for example, "building materials"), and therefore it is necessary to fulfil detailed requests to the equipment manufacturing enterprise about the possibility of a specific device for determining the content of closed cells in the polyurethane foam. The same situation with information on the cost of equipment. As a rule, instruments that determine the apparent density and porosity have such function and determine the number of closed cells (but not necessarily).
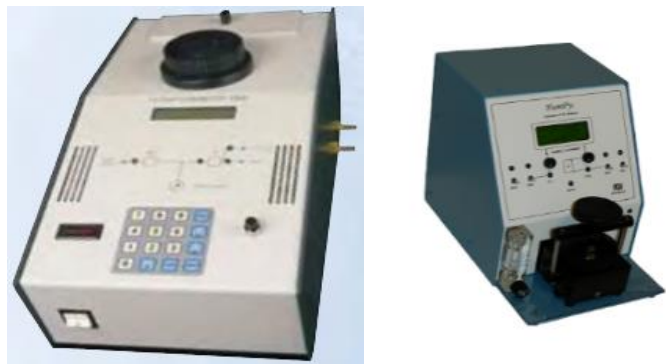

Fig. 4 - automatic pycnometers for determination of foam characteristics

On automatic pycnometers such as Pycnomatic, all operations, such as calibration, sample preparation, measurement and calculations, are automatically performed. The instrument automatically repeats the measurements until the specified accuracy is reached. The measurement takes 2-3 minutes. For example, in [2] (edition of 2017) it is stated that the most common device for these purposes is currently the American Ultrapyc 1200e manufactured by Quantachrome Corporation, USA. However, in the opinion of the authors, UltraFoam 1200e and PentaFoam 5200e are more suitable, since in the technical description there is a reference to the test methods for polyurethane foam. 


\section{Conclusion}

The parameter of the content of the number of closed cells for rigid polyurethane foam is an important characteristic that determines the durability of the material and its thermal insulation properties. Based on the analys is of test methods for determining the volume percentage of open and closed cells of rigid sprayed polyurethane foams and the resulted sequences of test procedures, it can be concluded that the principle of operation of a pycnometer based on ISO 4590 is generally more complex and time-consuming than the operation of a pycnometer based on ASTM D 6226, the structural details of the pycnometer are different. Both standards are quite controversial in accuracy, measurement errors and in the reproducibility of the result, for example, in a pycnometer for ISO 4590, pressure gauges may exhibit instability, as a result of which measurements is not made due to the presence of anomalies.

ISO Standard 4590 contains two methods for determining open cells: the method of changing pressure and the method of expanding the volume. The first method is similar to the method in ASTM D6226, but differs in the preparation of the experiment and in the number of samples.

For the developed Russian standard for rigid PUR and PIR foams [9], it is necessary to harmonize the ISO 4590 standard to determine the volume percentage of open and closed cells of rigid sprayed polyurethane foam, since the first method is similar to the method in ASTM D 6226, the method in ISO 4590 contains the classification according to groups the number of closed cells, which are further referred to European standards for polyurethane foam.

\section{References}

1. BS EN 14315-1:2013

2. G. Wypych. Handbook of Foaming and Blowing Agents. (ChemTec Publishing, Toronto, 2017)

3. A.G. Demenyeva, T.K. Khlystalova, A.I. Demina, P.A. Zinger. High-molecular compounds.33-10. 2257 - 2266 (1991)

4. Y. Zhang, S. Parent, M. Kontopoulou and C.B. Park. Journal of Cellular Plastics. 0(0) 1-18 (2015)

5. N.V Gama, B. Soares, C.SR Freire, R. Silva, A. Ferreira, , A. Barros-Timmons. Journal of cellular plastics. 1-18 (2017) https://doi.org/10.1177/0021955X17732304

6. ISO 4590

7. ASTM D6226-15

8. Pycnomatic gas (helium) pycnometer from Thermo Scientific.

9. M. Gravit, A. Kuleshin, E. Khametgalieva, I. Karakozova, IOP Conf. Ser.: Earth Environ. Sci. 90012187 (2017) 